Revue internationale de l'économie sociale

Recma

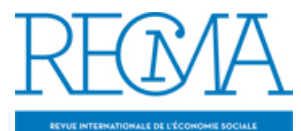

\title{
Bibliographie juridique en économie sociale
}

\section{David Hiez}

Numéro 306, octobre 2007

URI : https://id.erudit.org/iderudit/1021260ar

DOI : https://doi.org/10.7202/1021260ar

Aller au sommaire du numéro

\section{Éditeur(s)}

Association Recma

\section{ISSN}

1626-1682 (imprimé)

2261-2599 (numérique)

Découvrir la revue

\section{Citer ce document}

Hiez, D. (2007). Bibliographie juridique en économie sociale. Revue internationale de l'économie sociale, (306), 96-97.

https://doi.org/10.7202/1021260ar 


\title{
BIBLIOGRAPHIE JURIDIQUE EN ÉCONOMIE SOCIALE
}

\author{
par David Hiez
}

Cette revue bibliographique couvre la période de parution juillet 2006-juin 2007.

\section{Monographies}

Bérard Caroline, La place de la structure juridique coopérative dans les stratégies du secteur bancaire français, thèse Bordeaux-IV, 2006.

Bourreau-Dubois Cécile et Jeandidier Bruno (dir.), Economie sociale et droit (2 tomes), Journées de l'Association d'économie sociale (26 septembre 2006, Nancy), L'Harmattan, 2006.

Ferraton Cyrille, Associations et coopératives, une autre histoire économique, "Sociétés en changement ", Eres éd., 2007.

Joing Jean-Luc, La bonne gouvernance des associations, dynamiques d'entreprises, L'Harmattan, 2007. Larifla Karyne, L'entreprise associative: contribution au droit des groupements, thèse, université des Antilles et de la Guyane, 2006.

Lefils Bernard, Fiscalité des associations, Litec, 2007.

Richez-Battesti Nadine et Gianfaldoni Patrick, Les banques coopératives en France, le défi de la performance, "L'esprit économique", L'Harmattan, 2007.

Rogeaux Claude, La responsabilité pénale, civile et financière des associations et de leurs dirigeants, Voiron, Territorial éd., 2006.

Sévilla Nathalie, Fuchs Julien, Besse Laurent et Bridiez Alain, La démocratie associative: perspectives historiques, L'Harmattan, 2006.

\section{Articles et chroniques}

Barbieri Jean-Jacques, «A propos de l'exercice du droit de rétention par une coopérative ", Dr. rur., février 2007, comm. 36.

Barbieri Jean-Jacques, "Actualisation du statut de la coopération agricole ", Dr. rur., février 2007, étude 14.

Barbieri Jean-Jacques, "Modifications de l'exploitation et continuité de l'engagement de coopération ", Dr. rur., avril 2007, comm. 152. Barbieri Jean-Jacques, "Propriété réservée et coopérative en difficultés ", Dr. rur., novembre 2006, comm. 332.

Barbieri Jean-Jacques, Formage Christelle, "Sur les placements à risque par une coopérative agricole ", Dr. rur., octobre 2006, comm. 254. Bardon Cyrille, "La prise en charge par une commune des dettes d'une association culturelle transparente ", Droit administratif, octobre 2006, comm. 146.

Bazex Michel et Blazy Sophie, «L'application de la réglementation bancaire aux caisses de crédit municipal », Droit administratif, juillet 2006, comm. 125. Bouveresse Aude, "Bases juridiques autorisant la création d'organismes dotés d'une personnalité juridique propre (à propos de la SCE) ", Europe, juillet 2006, comm. 203.

Deboissy Florence et Wicker Guillaume, "L'association qui ne remplit plus l'objet qui était le sien peut être dissoute pour justes motifs ", JCP G, 2007, II, 10105.

Delacour Eric, "Contrat nouvel embauche et mieux-disant social ", (comm. TA Bordeaux, 5 décembre 2006), Contrats et marchés publics, avril 2007, comm. 101.

Eckert Gabriel, «Contrat conclu avec une association transparente et responsabilité administrative ", Contrat et Marché public, mai 2007, comm. 137.

Ferry Patrick, «Présentation de quelques dispositions de la loi ENL ", Construction Urbanisme, octobre 2006, étude 17. 
Gros Laurent, " Modernisation du statut des coopératives agricoles (les restructurations de coopératives agricoles) ", Dr. sociétés, janvier 2007, prat. 1.

Karpenschif Michaël, «De l'utilité d'une association transparente ", JCP A, 2007, 2111.

Lafond Jacques, "Loi du 13 juillet 2006 (dite loi ENL) et copropriété ", JCP N, 2006, 1282.

Le Vey Pierre, "L'évolution du droit des sociétés coopératives de production ouvrière (Scop) ", Dr. sociétés, octobre 2006, étude 18.

Lécuyer Hervé, «Personnalité des caisses régionales de crédit agricole sans immatriculation au RCS », Dr. sociétés, juillet 2006, comm. 102.

Lécuyer Hervé, "Sanction de l'abus dans une association et réflexion sur les critères de l'abus dans les sociétés ", Dr. sociétés, août 2006, comm. 119.

Lucas François-Xavier, "Pouvoirs d'un président d'association et application du droit des sociétés à une association ", JCP E, 2006, 2675. Lucas François-Xavier, " Exclusion d'un membre d'une association ", Dr. sociétés, avril 2007, comm. 66.

Lucas François-Xavier, «Liberté de se retirer d'une association ", Dr. sociétés, janvier 2007, comm. 4. Lucas François-Xavier, " Pouvoir des dirigeants de coopératives de conclure des opérations de gestion financière étrangère à l'activité principale de la société ", Dr. sociétés, octobre 2006, comm. 140.

Lucas François-Xavier, « Pouvoirs d'un président d'association ", Dr. sociétés, novembre 2006, comm. 158.
Moreau Jacques, « De la délicate appréciation de l'intérêt public local dans l'octroi de subventions à des associations ", JCP A, 2006, 1152.

Morvan Patrick, « Reprise d'entreprise en liquidation judiciaire par les salariés et absence de contrat en cours au sens de l'article L. 122-12 ", JCP S, 2007, 1134.

Pierre Jean-Luc, " Participation à des actions de développement de réseau d'entreprises (comm. CE, 6 mars 2006) ", Dr. sociétés, juillet 2006, comm. 117.

Rédaction, "Modernisation du statut des coopératives agricoles ", Dr. sociétés, 2006, prat. 7 .

Rédaction, "Adaptation des sociétés coopératives agricoles aux évolutions du droit des sociétés ", JCP E, 2006, act. 436.

Reifegerste Stéphane et Gaulier Aurélie, "Application au président du conseil d'administration d'une société membre d'un groupement coopératif de la clause compromissoire stipulée dans le règlement intérieur du groupement ", JCP E, 2007, 1268.

Roman-Séquense Brigitte, "Comment peut-on intégrer le commerce équitable dans les marchés publics ", Contrat et Marché public, octobre 2006, comm. 276.

Rossignol Jean-Luc, « Fiscalité et réseau de distribution Leclerc », Dr. fiscal, 2007, comm. 490. Thomas Vincent, «L'appel public à l'épargne des clubs sportifs ", JCP E, 2007, 1326.

Vigneron Guy, « Paiement des cotisations de l'association gestionnaire des services spécifiques ", Loyer et Copropriété, février 2007, comm. 38.

\begin{tabular}{|ll|}
\hline Abréviations & \\
Act. Prat. Ing. Soc. : Actes pratiques et Ingénie- & JCP A: La Semaine juridique Administrations et \\
rie sociétaire. & Collectivités territoriales. \\
AJFP: Actualité juridique Fonctions publiques. & JCP E: La Semaine juridique Entreprise et Affaires. \\
Bull. Joly: Bulletin Joly Sociétés, actualité du droit & JCP N: La Semaine juridique notariale et immo- \\
des sociétés. & bilière. \\
CCE: revue Communication Commerce élec- & PA: Petites Affiches, la loi, le quotidien juridique. \\
tronique. & RDSS: Revue de droit sanitaire et social. \\
D.: Dalloz. & Rev. Soc.: Revue des sociétés. \\
Dr. rur.: Revue de droit rural. & RTDcom.: Revue trimestrielle de droit commer- \\
Dr. sociétés: revue Droit des sociétés. & cial et de droit économique. \\
\hline
\end{tabular}

